\title{
Catégorisation des apprenants: une étude de la structure interne des cognitions des enseignants sur les élèves
}

\section{Lara Laflotte, Angela Mossaz, Marie-Lousie Aliprandi et Philippe Wanlin}

Cette contribution propose d'étudier la structure interne des cognitions des enseignants sur les élèves par l'intermédiaire de deux méthodes de catégorisation d'élèves: celle par descriptifs de types d'élèves et celle par regroupements d'élèves. A la lumière des théories relatives à l'organisation des cognitions, notre recherche, menée auprès de neuf enseignantes genevoises, montre une organisation typologique des cognitions des enseignants sur les élèves en clusters selon une structure centrale-périphérique. L'association aux prototypes de clusters n'étant pas toujours évidente pour certains élèves, nous rejoignons l'idée d'isolement entre les cognitions formulée par Green (1971) qui accepte une certaine perméabilité des frontières entre clusters.

\section{Introduction}

Pour de nombreux textes, les enseignants prennent leurs décisions pédagogiques en se basant sur les élèves. Que ce soit lors de la planification de leurs leçons (Wanlin, 2009) ou lorsqu'ils les donnent en classe (Wanlin \& Crahay, 2012), la littérature admet que les enseignants se réferent à un ou des groupe(s) d'élèves (Berliner, 1987; Bromme, 1989). Plus précisément, deux notions sont conjecturées par les chercheurs: le steering group (Dahllöf, 1971) - groupe d'élèves (faibles) que l'enseignant utilise comme référence pour cadencer son instruction - et le case knowledge (Calderhead, 1996) - heuristique(s), notamment d'élèves, disponible(s) dans le répertoire cognitif des enseignants pour décider. Hélas, la littérature nous renseigne peu sur la manière dont ces entités cognitives sont constituées.

Seul Hofer (1986) avance une explication de la structure des cognitions sur les élèves. Pour lui, elles sont composées de plusieurs catégories d'élèves aux frontières nettes organisées, à l'interne, en un noyau central autour duquel gravitent les élèves des différentes catégories sans que ceux-ci ne correspondent tout à fait au centroïde prototypique. Cette vision est hypothétique car Hofer ne l'a pas testée. 
Cette contribution propose d'explorer cette structure hypothétique par l'intermédiaire de méthodes utilisées pour étudier les cognitions des enseignants sur les élèves. Nous nous intéressons dans un premier temps à celles qui ont mis en évidence le recours à des catégories d'élèves. Elles nous permettront ensuite d'exposer brièvement les théories relatives à l'organisation des cognitions disponibles dans la littérature. Suite à la présentation de notre échantillon et de notre méthode de recherche, nous relaterons nos résultats que nous discuterons à la lumière des théories susmentionnées. Quelques pistes pour les recherches futures et la formation des enseignants seront alors suggérées.

\section{Cadre théorique}

\section{Les typologies d'élèves au sein du bagage cognitif des enseignants}

Nombre de chercheurs estiment que les enseignants regroupent les informations sur les élèves en des entités cognitives, utilisées sous la forme de catégories stéréotypées d'élèves (Berliner, 1987; Calderhead, 1983; Hofer, 1986; Mayer \& Marland, 1997). Ces entités cognitives sont des rassemblements d'élèves qui se ressemblent relativement aux informations condensées, et permettent aux enseignants d'enseigner (Bromme, 1989).

Les recherches qui s'intéressent aux catégories d'élèves utilisent notamment deux approches: par descriptifs de types d'élèves et par regroupements d'élèves.

\section{L'approche par descriptifs de types d'élèves}

Cette approche est utilisée par Thelen (1967) auprès de 70 sujets intervenants dans des écoles américaines. Ces sujets énumèrent par écrit des types d'élèves rencontrés dans les salles de classe du secondaire. Suite à une analyse du contenu, Thelen condense les 300 descriptions obtenues en 26 sous-types d'élèves qu'il classe en quatre ensembles: les «bons élèves» intéressés et volontaires, les «élèves indifférence» agréables, mais peu soucieux de l'éducation, les «mauvais élèves» agaçants et durs à gérer, et les "élèves perdus» tranquilles mais retirés.

Kagan et Tippins (1991) demandent à 12 futurs enseignants (cinq candidats du primaire et sept du secondaire) de leur remettre des descriptions de types d'élèves. En se basant sur leurs observations et interactions personnelles en classe et en dehors de celle-ci, les futurs enseignants sont invités à décrire ces types selon plusieurs variables au choix telles que: résultats et motivation scolaires, caractéristiques intellectuelles, personnalité, background familial, relations avec les camarades de classe ou l'enseignant, etc. Par une analyse du contenu, les chercheuses observent que $23 \%$ des descriptions des candidats du primaire mentionnent le type «élève modèle» et $77 \%$ le type «élève qui dérange, peu motivé et/ou possédant peu de compétences sociales». Pour ceux du secondaire, $24 \%$ des descriptions décrivent le type "élève modèle non problématique», 
$24 \%$ le type «élève peu motivé peu préoccupant» et, $52 \%$ le type «élève aux comportements non scolaires».

Hörstermann, Krolak-Schwerdt et Fischbach (2010) adoptent également une démarche par descriptions de types d'élèves tout en complétant la méthode d'analyse de contenu par une analyse en clusters. Quatre-vingt-deux étudiants luxembourgeois en 1 ère et $2^{\text {ème }}$ années de formation en enseignement primaire participent à cette étude. Pour procéder à la description des types d'élèves rencontrés, les étudiants proposent une étiquette pour décrire chaque type d'élèves et énumèrent leurs caractéristiques comme la personnalité, les comportements, l'environnement social, le statut socioéconomique, les compétences, etc. Après deux opérations de condensation successives réalisées lors d'une analyse de contenu manuelle, ce sont 20 types qui sont retenus par les chercheurs. Une triangulation des méthodes de clustering hiérarchiques agglomératives à partir des fréquences des caractéristiques descriptives des 20 types aboutit à la conservation de 10 ensembles. Il s'agit des élèves: modèles, vivants, «monsieurje-sais-tout», clowns, agressifs, hyperactifs, rêveurs, fainéants, «je-m'en-foutiste» et socialement retirés.

\section{L'approche par regroupements d'élèves}

Morine-Dershimer (1978) propose à 10 enseignants d'effectuer un tri d'élèves selon les critères de leur choix à cinq moments de l'année scolaire. A l'aide d'étiquettes avec le prénom de leurs élèves, ils rassemblent leurs élèves pour former des groupes selon une caractéristique à nommer. Ils peuvent former autant de groupes avec autant d'élèves qu'ils le souhaitent, et ce, à chaque moment de l'année.

Six ensembles de caractéristiques sont recueillies par la chercheuse: compétences et réussite, participation dans l'enseignement, personnalité, relations avec les pairs, orientation des activités, et développement et progrès. Au début, les enseignants sont centrés sur la personnalité de leurs élèves (23\% des catégories). Au fil de l'année, ils se focalisent sur leur participation en classe (22\%), puis, ils terminent l'année en privilégiant le progrès $(15,6 \%)$ et les relations entre pairs $(15,6 \%)$. La compétence de l'élève n'est jamais dominante (entre 3\% et 12,5\%) (Morine-Dershimer, 1978).

Les résultats de Morine-Dershimer (1978) mettent en évidence des critères d'évaluation des élèves au cours de l'année scolaire. Sans donner les étiquettes utilisées pour nommer les regroupements, ces résultats peuvent être rapprochés de ceux des recherches par descriptifs de types d'élèves puisque la motivation, les comportements, la personnalité et la sociabilité jouent un rôle central.

Une équipe toulousaine a utilisé une méthode similaire en remplaçant les étiquettes-prénoms par des photographies (Veyrac \& Blanc, 2014). Ces chercheuses s'intéressent aux caractéristiques des élèves retenues dans les discours de 67 enseignants de six établissements français du secondaire de six régions et identifient les règles d'action, les stratégies des enseignants en lien avec ces catégo- 
risations en termes de gestion de la classe. Veyrac et Blanc (2014) identifient des perceptions et dénominations aussi étendues que variées. Elles montrent qu'il y a différentes logiques de regroupement: une logique explicite, stable, relevant d'un critère précis, et une logique mobile, relevant de multicritères changeants qui fonde la plupart des regroupements. Elles observent que les élèves ne sont pas tous vus selon les mêmes indices perceptifs, donc régulièrement la logique des critères mobilisés par un même enseignant n'apparait pas de manière évidente, de même que la logique interne des sous-groupes. Elles constatent aussi que le processus de catégorisation varie en fonction de l'âge et de l'ancienneté des enseignants, que les préoccupations des enseignants tournent autour de l'élève dans la tâche scolaire, son engagement et sa performance et que les enseignants privilégieraient les élèves en difficulté au risque de sacrifier un groupe d'élèves plus avancés, même si ceux-ci peuvent être amenés à aider les autres élèves.

Ces approches présentées, nous observons l'absence de données sur la structure interne des cognitions des enseignants sur les élèves. Or, si nous voulons contribuer à l'apprentissage de ces cognitions, il nous faut connaître leur structure interne (Roussiau \& Bonardi, 2001). Les recherches qui décrivent une corrélation entre compétences diagnostiques et efficacité dans l'apprentissage des élèves (Hattie, 2012) n'étant pas suffisamment développées pour expliquer l'organisation interne des cognitions, nous présentons quelques théories pouvant nous avancer sur ce point.

\section{Théories descriptives de la structure interne des cognitions}

Les théories relatives à la catégorisation apportent un éclairage à propos de la structure des cognitions. On peut distinguer dans la littérature plusieurs théories dont deux principales (Reed, 2011).

La première est la théorie classique des conditions nécessaires et suffisantes (Hofer, 1986; Sternberg, 2007). Elle considère qu'une catégorie est définie en un ensemble présentant en tous points des caractéristiques identiques, tous les membres sont représentatifs de celle-ci et sont donc tous des représentants égaux et parfaits. Plus précisément, un élément donné ne peut appartenir à une catégorie que s'il possède l'ensemble des caractéristiques que renferme la catégorie en question. Dès lors, disposer de l'ensemble de ces caractéristiques suffit à l'élément pour être identifié à cette catégorie. S'il ne réunit pas l'ensemble des caractéristiques, il ne peut appartenir à cette catégorie. Par conséquent, chaque catégorie a des frontières délimitées strictes. Cette conception a été critiquée car son manque de flexibilité ne permet pas de rendre compte d'une grande majorité de catégories.

Ainsi, la théorie des prototypes (Rosch, 1978) défend l'idée que l'esprit humain manipule des pseudo-catégories, au sein desquelles les éléments sont regroupés selon des degrés de proximité. La construction de ces catégories aux frontières imperméables est basée sur des caractéristiques décrivant les modèles 
typiques des catégories. Ces prototypes abstraits ne correspondent à aucun exemple expérimenté; la catégorisation dépend uniquement des similitudes avec les prototypes générés et stockés en mémoire (Medin \& Rips, 2005). Tout comme la théorie précédente, à partir du moment où il y a une ressemblance importante entre plusieurs éléments, ils peuvent appartenir à la même catégorie sans appartenir aux autres; les frontières entre catégories sont donc hermétiques. Toutefois, l'idée de ressemblance implique ici un fonctionnement selon un degré de proximité: les éléments peuvent être plus ou moins proches de leur modèle typique, sans qu'il n'existe forcément de représentant parfait. Cet axe théorique suppose la hiérarchisation des caractéristiques (Reed, 2011). À ce sujet, Green (1971) apporte une certaine nuance; d'après lui les croyances sont organisées selon une structure centrale-périphérique mais ne sont pas interreliées. Plus précisément, les croyances s'agglutinent entre elles pour créer différents clusters aux frontières perméables par la présence de certaines croyances plus ou moins isolées au sein de chacun des clusters.

Face à ces théories, des approches alternatives ont vu le jour. Ainsi, contrairement à la théorie des prototypes, la théorie de l'exemplaire affirme la récupération d'informations en mémoire via la présence d'exemplaires stockés dans chaque catégorie. Il ne s'agit donc pas, comme dans la théorie précédente, de faire appel à une information abstraite, un prototype d'une catégorie, car tous les exemplaires sont archivés et accessibles (Medin \& Rips, 2005). Un nouvel objet pourra ainsi être classé et stocké en mémoire en fonction de sa ressemblance avec des exemplaires comparables déjà stockés. D’autres approches encore proposent principalement des variantes des deux approches que nous présentons ici, et insistent sur l'influence de la situation pour expliquer un recours à des combinaisons d'approches et/ou défendent l'idée d'un modèle mixte (Medin \& Rips, 2005).

Au regard d'une littérature dense et variée sur les théories relatives à la structure interne des cognitions, Medin et Rips (2005), mais aussi Murphy (2010), pointent toute la complexité des notions de concept et catégorie utilisés par tout être humain pour organiser et identifier le monde qui l'entoure. Toutefois, sur l'ensemble des travaux susmentionnés, seuls ceux de Hofer (1986) proposent une hypothétique structure pour les cognitions sur les élèves.

Par une analyse en clusters réalisée à partir des jugements d'enseignants sur leurs élèves, il obtient un système en cinq ensembles d'élèves structurés de manière centrale-périphérique (Hofer, 1981). Cette conclusion a été critiquée étant donné que le propre de l'analyse en clusters est de regrouper des objets par proximité et d'organiser les données de manière centrale-périphérique avec des frontières hermétiques (Oldenbürger, 1986).

Dans ce texte, nous proposons d'aller au-delà de l'analyse en clusters pour étudier la structure des cognitions enseignantes sur les élèves. Pour ce faire, nous rejoignons les démarches méthodologiques engagées dans de nombreuses recherches en psychologie sociale pour étudier les représentations sociales. À la 
manière d'Abric (2003), nous définissons les cognitions enseignantes comme un système socio-cognitif intégrant une organisation spécifique autour d'un noyau central. De ce fait, tout comme Abric avec les représentations sociales, nous étudions les cognitions enseignantes sur les élèves en deux temps. Après avoir produit un maximum de mots/expressions pour décrire les groupes/types d'élèves rencontrés, nous demandons aux enseignantes, en plus de hiérarchiser ces mots au sein de chacun des groupes/types d'élèves, de classifier leurs élèves selon leur degré de typicalité au sein de ces ensembles. Nous vérifions ensuite si l'analyse en clusters d'élèves correspond aux ensembles créés par les enseignantes et si leur organisation interne (en termes de typicité) coïncide. Cette méthode nous permettra de vérifier si la théorie des prototypes est applicable à ces cognitions.

\section{Mé thode}

\section{Public}

Neuf enseignantes de neuf écoles primaires de Genève ont participé à notre recherche. Elles ont entre 6 et 31 ans d'expérience $(\mathrm{m}=16,5)$ et exercent dans tous les degrés de l'enseignement primaire. La taille des différentes classes varie entre 17 et 23 élèves $(=20)$. L'expérience et les degrés d'enseignement ont un impact très limité sur les résultats.

\section{Études et tâches}

Notre recherche comporte deux études organisées sous forme d'interviews semi-dirigées intégrant chacune quatre tâches à destination des enseignantes.

Pour la première étude, la première tâche consiste à répliquer l'approche par descriptifs de types d'élèves (Hörstermann et al., 2010; Kagan \& Tippins, 1991; Thelen, 1967). Les enseignantes sont invitées à énumérer en moins de 10 minutes, différents types d'élèves et à les décrire à l'aide d'expressions sur des fiches (tâche assimilable à l'association libre de mots selon Abric, 2003). Ensuite, la deuxième tâche consiste à demander à chaque enseignante quel poids elle attribue à ces expressions afin de permettre la prise en compte de la hiérarchisation des caractéristiques descriptives. Pour vérifier la perméabilité des frontières entre les catégories, il s'agit, dans la troisième tâche, d'estimer les distances entre chaque type d'élèves. Enfin, l'objectif de la quatrième tâche est de vérifier les degrés de proximité aux centrö̈des et de renforcer les observations quant à la perméabilité des frontières des catégories: les enseignantes attribuent un type à chaque élève, et estiment, pour chacun, la distance d'appartenance à tous les types mentionnés. Ces dernières tâches peuvent être rapprochées au temps de hiérarchisation décrit par Abric (2003).

La deuxième étude est construite sur le même schéma, à la différence que l'approche proposée lors de la première tâche consiste à répliquer celle de 
Morine-Dershimer (1978) basée sur les regroupements d'élèves. Le tableau 1 présente l'ensemble des tâches.

\section{Tableau 1: Consignes données aux enseignantes pour chaque tâche en fonction des études}

Etude 1: descriptifs d'élèves Etude 2: regroupements d'élèves

\begin{tabular}{|c|c|c|}
\hline Tâche 1 & $\begin{array}{l}\text { Selon vous, durant votre } \\
\text { carrière, avez-vous rencontré } \\
\text { plusieurs types d'élèves } \\
\quad \text { différents? } \\
\text { Quels sont-ils? Pourriez-vous } \\
\text { les décrire à l'aide de } \\
\text { quelques expressions? }\end{array}$ & $\begin{array}{l}\text { Voici des étiquettes sur lesquelles sont notés les prénoms } \\
\text { de vos élèves. Pourriez-vous les classer pour regrouper les } \\
\text { élèves qui se ressemblent selon un ou des critères de votre } \\
\quad \text { choix. } \\
\text { Pour chacun des groupes d'élèves composés, expliquez en } \\
\text { quoi les élèves qui les constituent se ressemblent et en quoi } \\
\text { ils ne ressemblent pas aux élèves des autres groupes. }\end{array}$ \\
\hline Tâche 2 & \multicolumn{2}{|c|}{$\begin{array}{l}\text { Quel est le poids de l'expression pour décrire le type/groupe concerné? } \\
\text { Se prononcer sur un continuum allant de } 0 \text { (peu de poids) à } 10 \text { (beaucoup de poids). }\end{array}$} \\
\hline he 3 & \multicolumn{2}{|c|}{$\begin{array}{l}\text { Sur une échelle allant de } 0 \text { (superposé/proche) à } 10 \text { (très éloigné), dans quelle mesure un } \\
\text { type/groupe particulier est-il proche des autres types/groupes? }\end{array}$} \\
\hline \multirow[b]{2}{*}{ Tâche 4} & $\begin{array}{l}\text { Attribuez un type à chaque } \\
\text { élève. }\end{array}$ & \\
\hline & \multicolumn{2}{|c|}{$\begin{array}{c}\text { Estimez la distance d'appartenance de l'élève au type/groupe: } 0 \text { (représente à 100\%) à } 10 \\
\text { (très éloigné); puis, estimez, selon la même échelle, la distance de l'élève aux types/groupes } \\
\text { auxquels il n'a pas été associé. }\end{array}$} \\
\hline
\end{tabular}

\section{Traitements des données}

Les données des deux études sont identiques, c'est pourquoi nous les traitons de la même manière pour toutes les tâches.

Les fiches descriptives ou descriptions des groupes issues de la tâche 1 ont fait l'objet d'une analyse de contenu (Bardin, 1977; Richards, 2005). Les expressions sont classées à l'intérieur de cinq champs sémantiques, et ventilées selon leur valeur: positive ou négative (voir tableau 2). ${ }^{1}$ Comparativement à Hörstermann et al. (2010), nous n'attribuons pas la même valeur à toutes les expressions utilisées par les enseignantes mais pondérons les différentes expressions en fonction de l'importance descriptive attribuée par les enseignantes grâce aux poids donnés lors de la tâche 2 .

Notre analyse de contenu aboutit, pour chaque type ou groupement, à la conservation de cinq scores de caractéristiques (un par champ sémantique) pouvant adopter une valeur positive ou une valeur négative. Ces scores sont soumis à une analyse en clusters afin d'examiner si les types ou regroupements donnés par les enseignantes peuvent être résumés en des agglomérats distincts mais contenant des ensembles homogènes. Tout comme Hörstermann et al. (2010), nous avons procédé à une analyse en clusters par multiples méthodes hiérarchiques (Clatworthy, Buick, Hankins, Weinman \& Horne, 2005; Everitt, Landau, Leese \& Stahl, 2011). 
Tableau 2: Exemple d'expressions utilisées par les enseignantes selon leur champ sémantique et leur valeur

\begin{tabular}{ccc} 
Champs sémantiques & Valeur & Expressions \\
\hline \multirow{2}{*}{ Comportement } & Positive & Calme, discret, effacé \\
& Négative & Perturbateur, indiscipliné, bavard, hyperactif, agressif \\
\multirow{2}{*}{ Attention } & Positive & Intéressé, attentif, concentré \\
& Négative & Manque de concentration, dans la lune, s'ennuie \\
Personnalité & Positive & Sûr de lui, sociable, leader, attentionné, patient, gentil \\
& Négative & Manque de confiance, introverti, transparent \\
Compréhension & Positive & Se donne de la peine, moyen \\
& Négative & Besoin de différenciation, difficultés d'apprentissage \\
Application & Positive & Scolaire, volontaire, consciencieux, travailleur \\
& Négative & Inactif, à encourager \\
\hline
\end{tabular}

Les ensembles de profils ainsi obtenus sont étudiés grâce aux proximités ressenties par les enseignantes. Les distances de la tâche 3 permettent de situer les différents types ou groupes entre eux grâce à une technique d'échelonnement multidimensionnel (Everitt et al., 2011). Cette tâche autorise la vérification de l'étanchéité des frontières entre les catégories d'élèves qu'elles soient issues des études basées sur les descriptions de types ou sur les regroupements d'élèves. Les proximités obtenues en tâche 4 permettent de vérifier si les enseignantes estiment que les élèves sont proches ou non de l'ensemble auquel ils sont associés. Il s'agit d'examiner si les ensembles adoptent une organisation centrale-périphérique et si les frontières entre les ensembles sont poreuses ou hermétiques. Des analyses de variances ainsi que des statistiques descriptives simples permettent d'étudier ces questions.

\section{Résultats}

\section{Étude 1}

\section{Catégorisation des types d'élèves (tâches 1 \& 2)}

Après suppression de cinq types spontanés dans lesquels les enseignantes n'ont classé aucun élève lors de la tâche 4, nous avons 45 types d'élèves. Nous utilisons, dans SPSS 22, la méthode de classification agglomérative de Ward (figure 1) sur les scores normalisés des champs sémantiques en prenant le carré de la distance euclidienne comme mesure de proximité (Everitt et al., 2011). 


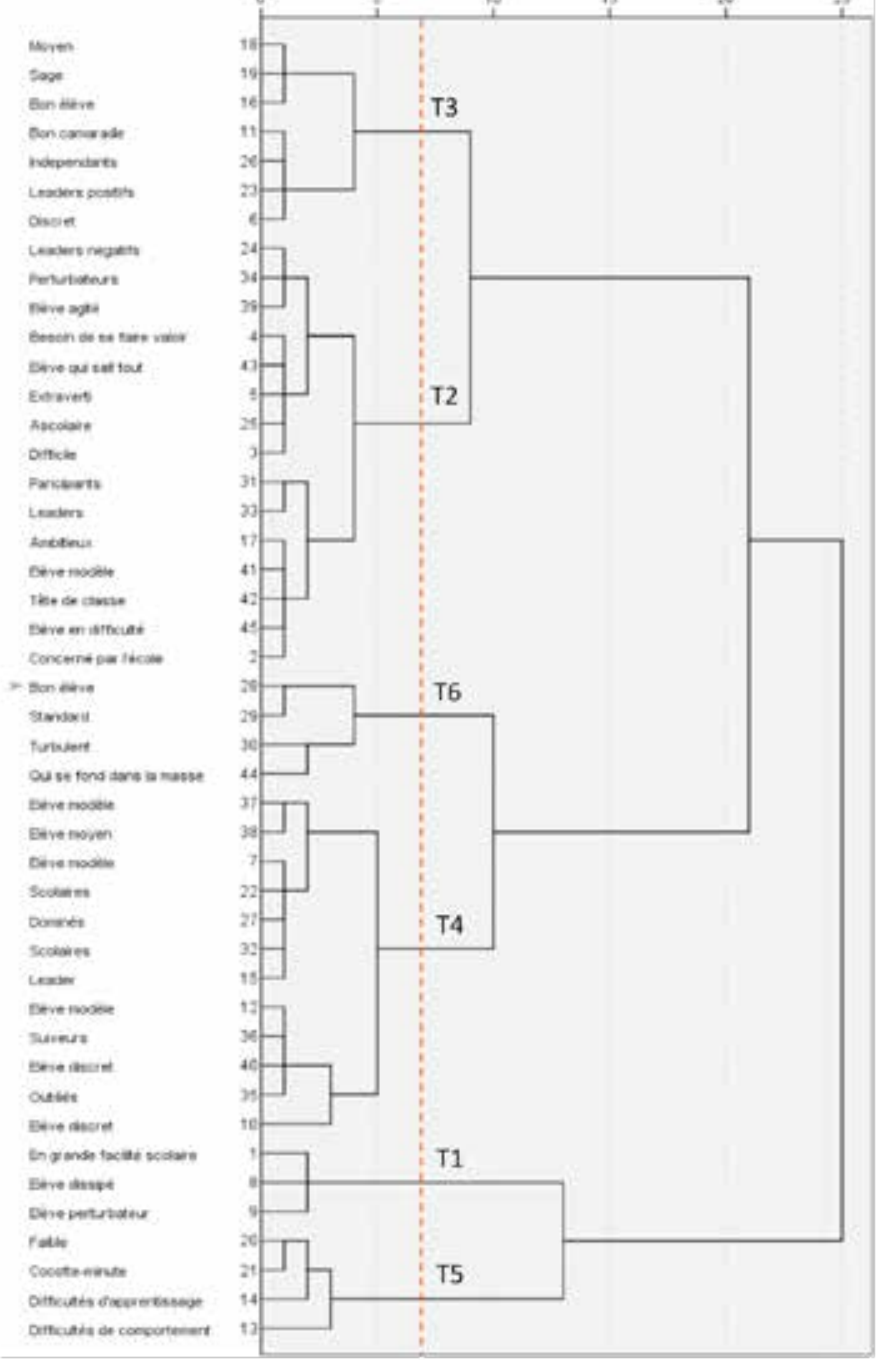

Figure 1: Dendrogramme d'agglomération des types d'élèves (Ward)

Selon le dendrogramme, nous obtenons six clusters. Pour vérifier ce choix, nous avons soumis les données à une analyse en nuées dynamiques qui aboutit à une solution similaire dans $58 \%$ des cas avec un recouvrement significatif

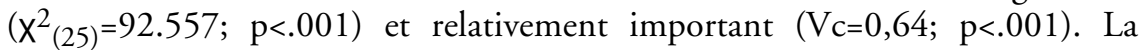
comparaison des moyennes obtenues aux scores de caractéristiques (tableau 3) permet de décrire chacun des ensembles de types spontanés. 
Tableau 3: Comparaison des moyennes aux scores de caractéristiques selon les clusters (moyenne, écart-type, ANOVA et test post hoc)

\begin{tabular}{cccccccccc} 
Variables & T1 & T2 & T3 & T4 & T5 & T6 & $\mathbf{F}_{5 / 39}$ & sig. & Scheffé \\
\hline $\begin{array}{c}\text { Compor- } \\
\text { tement }\end{array}$ & $-0,4$ & $-0,44$ & 0,54 & 0,53 & $-0,01$ & $-0,01$ & & & \\
& $(0,44)$ & $(0,31)$ & $(0,32)$ & $(0,34)$ & $(0,72)$ & $(0,69)$ & 10,31 & .000 & {$[(2=1)<(4=3)]=(6=5)$} \\
Attention & $-0,57$ & 0,54 & 0,40 & 0,45 & $-0,63$ & 0,18 & 1,68 & .000 & $(5=1)<(6=3=4=2)$ \\
& $(0,45)$ & $(0,30)$ & $(0,26)$ & $(0,36)$ & $(0,12)$ & $(0,48)$ & & & \\
Person- & 0,27 & 0,29 & 0,48 & $-0,19$ & $-0,46$ & $-0,01$ & 12,25 & .000 & $(5<3)=\{[4<(2=1)]=6\}$ \\
nalité & $(0,25)$ & $(0,24)$ & $(0,12)$ & $(0,30)$ & $(0,41)$ & $(0,03)$ & & & \\
Compré- & $-0,90$ & $-0,05$ & $-0,06$ & $-0,07$ & 0,00 & 0,64 & 32,81 & .000 & $1<(4=3=2=5)<6$ \\
hension & $(0,17)$ & $(0,12)$ & $(0.09)$ & $(0,23)$ & $(0,00)$ & $(0,15)$ & & & \\
Appli- & 0,21 & 0,18 & 0,04 & 0,76 & $-0,48$ & 0,46 & 14,86 & .000 & $(3=) 5<4[=6=1=2(=3)]$ \\
cation & $(0,22)$ & $(0,22)$ & $(0,45)$ & $(0,21)$ & $(0,09)$ & $(0,41)$ & & & \\
\hline
\end{tabular}

Nous observons deux ensembles de types perturbateurs qui adoptent une orientation différente en termes de compréhension et d'attention: les perturbateurs attentifs relativement bons élèves (cluster T2) et les perturbateurs inattentifs peu performants (T1). Proches de T2 en ce qui concerne la variable compréhension, deux ensembles de types d'élèves calmes sont représentés: les calmes attentifs et attentionnés (T3) qui se distinguent par leur score de personnalité, et les calmes attentifs et scolaires (T4) qui se caractérisent par leur score d'application. Malgré un score de compréhension moyen, le cluster $\mathrm{T} 5$ réunit des types d'élèves avec des difficultés dans l'application, la personnalité et l'attention; il s'agit des élèves moyens inattentifs introvertis et à encourager. Enfin, le cluster T6 obtient des scores relativement positifs à l'ensemble des variables et tout particulièrement à celle de la compréhension, on peut donc le nommer les bons élèves scolaires et attentifs.

\section{Proximités et distances perçues entre les types d'élèves (tâche 3)}

Nous avons procédé à une analyse en échelonnement multidimensionnel sur les proximités moyennes des types émises par les enseignantes en fonction de leur cluster d'appartenance. Cette procédure statistique permet de positionner des objets dans un plan lorsqu'on connait les distances qui les séparent (Everitt et al., 2011).

L'algorithme d'échelonnement multidimensionnel place les différents clusters sur un plan à deux dimensions (figure 2), composé de plusieurs zones délimitées par deux composantes. La première composante (traits doubles) est relative au comportement: elle différencie les clusters selon la nature du comportement en classe d'après les descriptifs des enseignantes. Le trait double continu est la limite entre comportement acceptable et non acceptable (T1 et T2); le trait double discontinu délimite les descriptions différenciant un comportement standard (T5 et T6) d'un comportement positif (T3 et T4). 


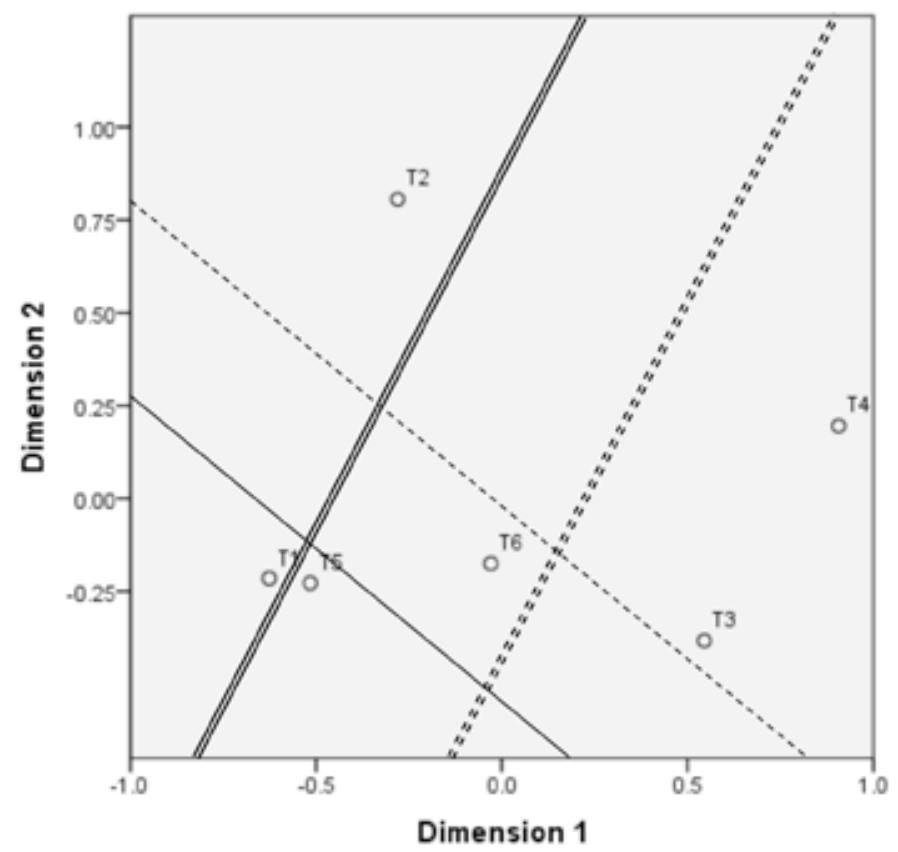

Figure 2: Position des clusters dans un plan bidimensionnel calculée à partir des distances inter-types estimées par nos enseignantes (échelonnement multidimensionnel)

La deuxième composante est relative à l'attention, la participation et la motivation (traits simples). En dessous du trait plein, il y a une zone dans laquelle sont situés des clusters aux faibles scores attentionnels (T1 et T5) et, au-dessus du trait discontinu, sont rangés des clusters aux descriptions positives en matière d'attention en classe (T2, T3 et T4). Entre, une zone tampon place le cluster obtenant des descriptions moyennes (T6).

L'interprétation du schéma bidimensionnel en zones délimitées par des composantes attentionnelle et comportementale, repose sur les définitions des clusters conçues à partir des descriptions des enseignantes et la comparaison de leurs moyennes aux scores de caractéristiques (tâches 1 et 2). La structuration du plan bidimensionnel repose, quant à elle, sur les estimations des proximités entre les types d'élèves perçues par les enseignantes (tâche 3). À partir de ces différentes données, nous obtenons des résultats superposables.

\section{Proximités-distances des élèves aux clusters de types (tâche 4)}

Hormis pour les clusters T5 et T6, le tableau 4 montre que les distances sont plus petites pour les clusters dans lesquels les élèves sont catégorisés que pour ceux dans lesquels ils ne le sont pas. 
Tableau 4: Comparaison des distances des élèves au cluster attribué et aux autres clusters (moyenne, écart-type, ANOVA et test post hoc)

Clusters et effectifs

\begin{tabular}{ccccccccccc}
\hline Distance & $\begin{array}{c}\text { T1 } \\
(\mathbf{n}=\mathbf{9})\end{array}$ & $\begin{array}{c}\mathbf{T} 2 \\
(\mathbf{n}=\mathbf{5 1})\end{array}$ & $\begin{array}{c}\text { T3 } \\
(\mathbf{n}=\mathbf{2 7})\end{array}$ & $\begin{array}{c}\mathbf{T} 4 \\
(\mathbf{n}=53)\end{array}$ & $\begin{array}{c}\text { T5 } \\
(\mathbf{n}=\mathbf{2 1})\end{array}$ & $\begin{array}{c}\text { T6 } \\
(\mathbf{n}=\mathbf{1 9})\end{array}$ & $\mathbf{F}$ & dll & Sig. & Scheffé \\
\hline $\begin{array}{c}\text { Au type } \\
\text { attribué }\end{array}$ & 2,11 & 4,06 & 2,11 & 3,94 & 5,43 & 4,12 & & & & $(1=3)<(6=5)$ \\
$(1,85)$ & $(2,18)$ & $(1,78)$ & $(1,85)$ & $(1,77)$ & $(1,62)$ & 9,03 & $1 / 174$ & .000 & $\begin{array}{c}(4=2)=(1=3) \\
(4=2)=(6=5)\end{array}$ \\
$\begin{array}{c}\text { Aux } \\
\text { autres }\end{array}$ & 7,02 & 6,32 & 6,06 & 7,24 & 6,13 & 5,5 & & & & \\
types & $(1,51)$ & $(2,32)$ & $(1,35)$ & $(2,09)$ & $(1,44)$ & $(0,66)$ & 2,25 & $5 / 158$ & .052 & $/$ \\
$\quad$ F & 37,97 & 25,94 & 84,67 & 73,96 & 1,97 & 2,05 & & & & \\
dll & $1 / 16$ & $1 / 100$ & $1 / 52$ & $1 / 104$ & $1 / 40$ & $1 / 20$ & & & & \\
Sig. & .000 & .000 & .000 & .000 & .169 & .168 & & & & \\
\hline
\end{tabular}

Ces statistiques rendent compte d'une organisation interne des cognitions selon la théorie de Green au moins pour quatre de nos ensembles de types: une structure centrale-périphérique avec isolement des cognitions via l'adoption d'une structuration en clusters non hermétiques.

Pour confirmer cette structure, des conditions doivent être rassemblées (Howell, 1998):

- les données ne sont pas distribuées normalement (significativité du test de Shapiro-Wilk en dessous de 0,05);

- les valeurs d'asymétrie doivent être:

- pour les distances aux clusters attribués: clairement (valeur absolue dépassant le double de l'erreur standard) négatives (distribution excentrée à gauche tel un L signifiant que les élèves du cluster sont proches);

- pour les distances aux clusters non attribués: clairement positives (distribution excentrée à droite tel un $\mathrm{J}$ signifiant que les élèves sont éloignés des autres clusters);

- les valeurs d'aplatissement doivent être clairement positives (la distribution a un pic élevé);

- les écart-types sont de petite taille indiquant que la distribution est resserrée autour de sa moyenne.

Le tableau 5 indique que les données sont normales pour toutes les distributions hormis celles de la distance au cluster attribué de T3 et des distances aux autres clusters pour T2 et T4. Dans tous les cas, les valeurs d'asymétrie et d'aplatissement se situent à l'intérieur de l'intervalle défini par le double de leur erreur standard, ce qui signifie que même si les distributions ne sont pas normales pour les clusters susmentionnés, on ne peut pas considérer qu'elles soient clairement asymétriques de l'un ou l'autre côté de la moyenne et qu'elles ont un sommet élevé (au contraire, nos données adoptent une distribution plutôt aplatie). Enfin, les écart-types adoptent des valeurs relativement élevées indiquant que les distributions s'écartent de leurs moyennes. 
Tableau 5: Statistiques descriptives et tests de normalité des distributions pour les distances aux clusters

\begin{tabular}{|c|c|c|c|c|c|c|c|c|c|c|c|c|}
\hline & & \multicolumn{8}{|c|}{ Statistiques descriptives } & \multicolumn{3}{|c|}{ Tests de normalité } \\
\hline & & Moy & E-t & Min & Max & Asym & $\begin{array}{c}\text { Er-Stand } \\
\text { (asym) }\end{array}$ & Aplat & $\begin{array}{l}\text { Er-Stand } \\
\text { (aplat) }\end{array}$ & $\begin{array}{l}\text { Shapi- } \\
\text { ro-Wilk }\end{array}$ & ddl & Sig \\
\hline \multirow{6}{*}{$\begin{array}{l}\text { Distances } \\
\text { aux } \\
\text { clusters } \\
\text { attribués }\end{array}$} & $\mathrm{T} 1$ & 2,11 & 1,85 & 0,0 & 5,5 & 0,63 & 0,72 & $-0,27$ & 1,4 & 0,940 & 9 & 0,581 \\
\hline & $\mathrm{T} 2$ & 4,06 & 2,18 & 0,0 & 8,3 & $-0,30$ & 0,33 & $-0,78$ & 0,66 & 0,962 & 51 & 0,101 \\
\hline & T3 & 2,11 & 1,77 & 0,0 & 6,3 & 0,57 & 0,45 & $-0,12$ & 0,87 & 0,907 & 27 & 0,019 \\
\hline & $\mathrm{T} 4$ & 3,94 & 1,85 & 0,0 & 8,0 & 0,00 & 0,33 & $-0,63$ & 0,64 & 0,977 & 53 & 0,412 \\
\hline & T5 & 5,42 & 1,77 & 2,5 & 9,0 & 0,33 & 0,50 & $-0,54$ & 0,97 & 0,975 & 21 & 0,843 \\
\hline & T6 & 3,33 & 4,16 & 0,0 & 8,0 & 1,29 & 1,22 & - & - & 0,923 & 3 & 0,463 \\
\hline \multirow{6}{*}{$\begin{array}{l}\text { Distances } \\
\text { aux autres } \\
\text { clusters }\end{array}$} & $\mathrm{T} 1$ & 7,02 & 1,51 & 5,0 & 9,3 & 0,26 & 0,72 & $-1,57$ & 1,4 & 0,926 & 9 & 0,446 \\
\hline & T2 & 6,32 & 2,32 & 0,0 & 10,0 & $-0,66$ & 0,33 & 0,38 & 0,66 & 0,942 & 51 & 0,014 \\
\hline & T3 & 6,06 & 1,35 & 3,5 & 9,0 & 0,08 & 0,45 & $-0,18$ & 0,87 & 0,980 & 27 & 0,856 \\
\hline & T4 & 7,24 & 2,09 & 3,0 & 10,0 & $-0,43$ & 0,33 & $-0,76$ & 0,64 & 0,939 & 53 & 0,009 \\
\hline & T5 & 6,13 & 1,44 & 3,5 & 9,0 & 0,52 & 0,50 & 0,11 & 0,97 & 0,947 & 21 & 0,293 \\
\hline & T6 & 5,50 & 0,66 & 5,0 & 6,3 & 1,46 & 1,23 & - & - & 0,893 & 3 & 0,363 \\
\hline
\end{tabular}

L'organisation centrale-périphérique est donc plausible car, globalement, les enseignantes attribuent des distances plus courtes aux élèves qu'elles associent à un cluster donné et des distances plus longues aux clusters auxquels elles ne les associent pas. D'autre part, les écarts entre les valeurs maximales et minimales ainsi que les indices d'asymétrie et d'aplatissement indiquent que les distances varient fortement d'un élève à l'autre. Quand certains élèves sont fort proches de leur cluster et fort éloignés des autres, d'autres obtiennent des distances intermédiaires se situant à l'intersection de plusieurs clusters. Les frontières entre clusters ne seraient donc pas hermétiques pour certains élèves.

Enfin, concernant l'idée de prototype (Rosch, 1978), les minimas des distances au cluster attribué indiquent que les clusters T1, T2, T3, T4 et T6 ont chacun au moins un élève qui obtient la distance de 0 au cluster. Ils correspondraient donc aux prototypes exemplaires des clusters considérés.

\section{Étude 2}

\section{Catégorisation des groupes d'élèves (tâches 1 \& 2)}

Pour ce clustering, nous avons utilisé la même méthode que pour l'étude 1. Le dendrogramme (figure 3) propose une solution à quatre clusters. Celle-ci a été confrontée à un algorithme divisif en nuées dynamiques pour aboutir à un recouvrement significatif $\left(\mathrm{x}^{2}{ }_{(9)}=71.085 ; \mathrm{p}<.001\right)$ et fort $\left(\mathrm{V}_{\mathrm{c}}=0,79 ; \mathrm{p}<.001\right)$ de $81,6 \%$. 


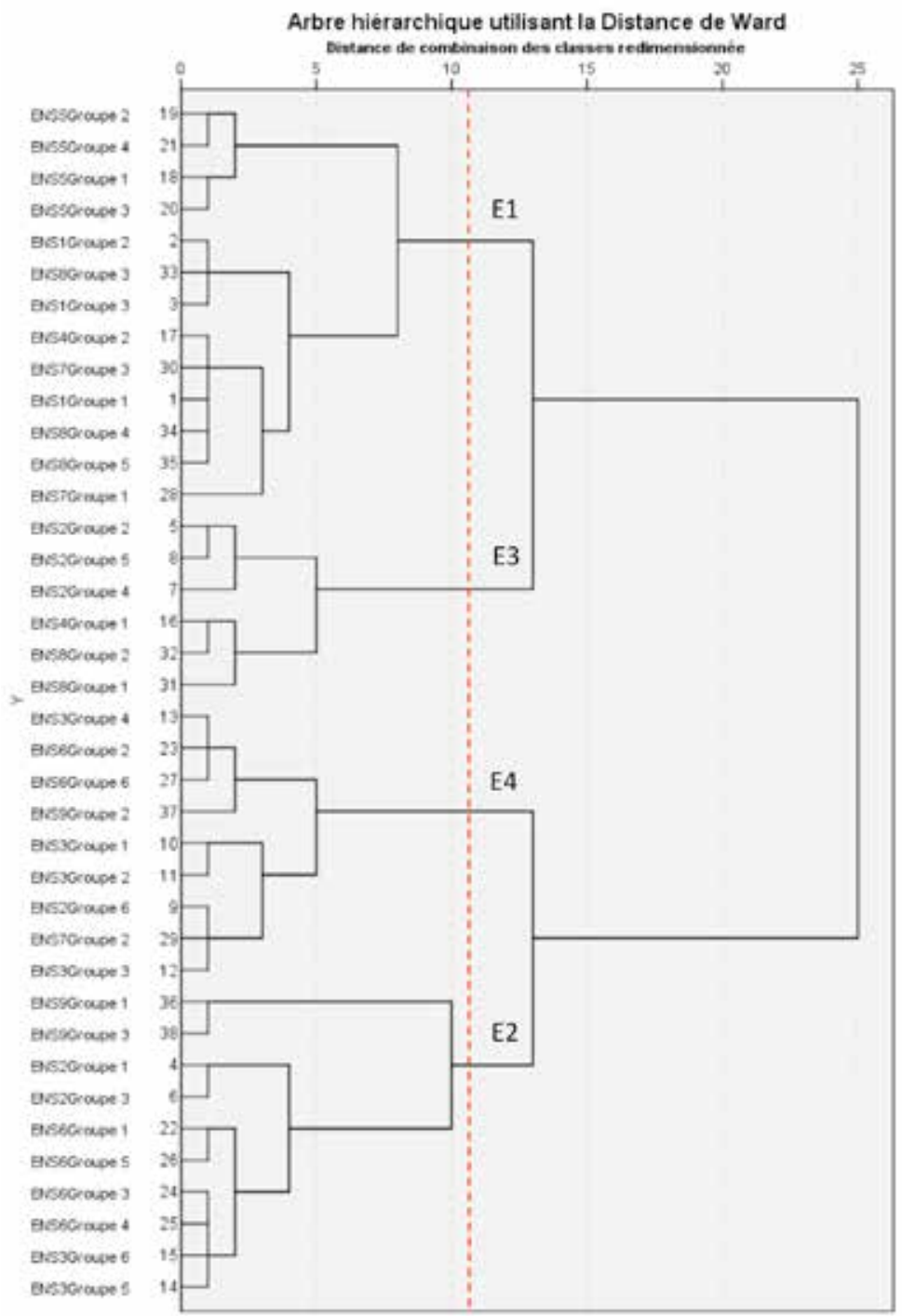

Figure 3: Dendrogramme d'agglomération des groupes d'élèves (Ward)

Les analyses de variance et les tests post-hoc fournis dans le tableau 6 permettent de décrire les quatre groupes obtenus.

$\mathrm{Au}$ vu des données, nous nommerons les clusters E1, les bons élèves peu consciencieux, E2, les agités peu performants, E3, les bons élèves volontaires, et E4, les élèves moyens discrets peu confiants. 
Tableau 6: Comparaison des moyennes aux scores de caractéristiques selon les clusters (moyenne, écart-type, ANOVA et test post hoc)

\begin{tabular}{cccccccc} 
Variables & E1 & E2 & E3 & E4 & $\mathbf{F}_{\mathbf{3} / \mathbf{3 4}}$ & Sig. & Scheffé .05 /.10 \\
\hline \multirow{2}{*}{ Comportement } & $-0,08$ & $-21,30$ & $-7,50$ & $-2,22$ & & & \\
& $(9,06)$ & $(10,40)$ & $(7,64)$ & $(5,38)$ & 13,11 & .000 & $2<(3=4=1)$ \\
Attention & $-2,23$ & 8,50 & 0,33 & 2,33 & 3,56 & .024 & $/$ \\
& $(4,62)$ & $(10,59)$ & $(5,47)$ & $(9,54)$ & & & \\
Personnalité & 2,15 & $-8,00$ & 9,67 & $-18,11$ & 17,91 & .000 & $(2=) 4<3(=1=2)$ \\
& $(7,50)$ & $(5,25)$ & $(13,32)$ & $(7,46)$ & & & \\
Compréhension & 5,46 & $-7,40$ & 4,17 & $-2,33$ & 7,48 & .001 & $(4=) 2<(3=1)(=4)$ \\
& $(0,09)$ & $(6,04)$ & $(6,21)$ & $(4,12)$ & & & \\
Application & 0,08 & 2,20 & 14,17 & 7,22 & 14,18 & .000 & $(2=) 1<4(=2)<3$ \\
& $(4,40)$ & $(4,92)$ & $(5,46)$ & $(4,29)$ & & & \\
\hline
\end{tabular}

Proximités et distances perçues entre les groupes d'élèves (tâche 3)

La figure 4 présente une analyse en échelonnement multidimensionnel sur les proximités moyennes des clusters de groupes données par les enseignantes selon leur cluster d'appartenance.

Nous observons des zones compartimentées en deux composantes. La première renvoie aux résultats scolaires; le trait pointillé simple indique le passage d'une performance moyenne à une performance positive. La deuxième zone représente l'application; les traits pointillés doubles délimitent l'application moyenne vers l'application élevée. Les constats sont similaires à ceux de l'étude 1 .

\section{Proximités-distances des élèves aux clusters de groupes (tâche 4)}

Le tableau 7 montre que les distances aux clusters d'appartenance sont plus resserrées que les distances aux autres clusters. Les distances aux clusters d'appartenance sont plus compactes pour le cluster E3 et plus étendues pour le cluster E1. Les éloignements aux autres clusters sont plus étendus pour le cluster E4 et plus étroits pour le cluster E1. Comme précédemment, au sein d'une organisation centrale-périphérique qui reste plausible dans la majorité des cas, certains élèves peuvent adopter des profils multidimensionnels à l'intersection de plusieurs catégories. L'observation du tableau 8 apporte les mêmes conclusions que précédemment: les frontières entre les catégories ne sont pas hermétiques. 


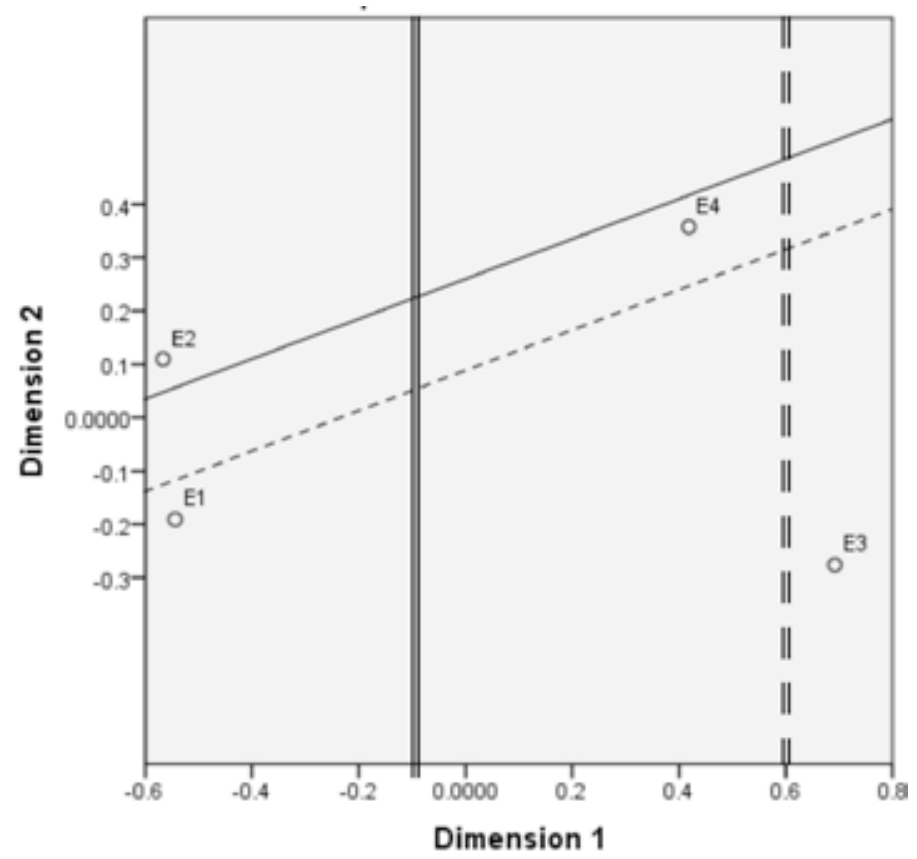

Figure 4: Position des clusters dans un plan bidimensionnel calculée à partir des distances inter-groupes estimées par nos enseignantes (échelonnement multidimensionnel)

Tableau 7: Comparaison des distances des élèves au cluster attribué et aux autres clusters (moyenne, écart-type, ANOVA et test post hoc)

\begin{tabular}{|c|c|c|c|c|c|c|c|c|}
\hline Distance & $\begin{array}{c}\mathbf{E 1} \\
(\mathbf{n}=77)\end{array}$ & $\begin{array}{c}\mathbf{E} 2 \\
(\mathbf{n}=45)\end{array}$ & $\begin{array}{c}\text { E3 } \\
(\mathbf{n}=30)\end{array}$ & $\begin{array}{c}\mathrm{E} 4 \\
(\mathrm{n}=29) \\
\end{array}$ & F & dll & sig. & Scheffe \\
\hline $\begin{array}{l}\text { Au groupe } \\
\text { d'apparte- } \\
\text { nance }\end{array}$ & $\begin{array}{c}4,84 \\
(1,59)\end{array}$ & $\begin{array}{c}4,07 \\
(1,64)\end{array}$ & $\begin{array}{c}3,23 \\
(1,33)\end{array}$ & $\begin{array}{c}3,97 \\
(2,29)\end{array}$ & 7,13 & $3 / 177$ & .000 & $\begin{array}{c}(4=2=) \\
3<1 \\
(=2=4)\end{array}$ \\
\hline $\begin{array}{l}\text { Aux autres } \\
\text { groupes }\end{array}$ & $\begin{array}{c}6,53 \\
(2,49)\end{array}$ & $\begin{array}{c}7,24 \\
(1,67)\end{array}$ & $\begin{array}{c}7,74 \\
(1,69)\end{array}$ & $\begin{array}{c}8,52 \\
(1,88)\end{array}$ & 6,09 & $3 / 140$ & .001 & $\begin{array}{l}(2=3=) \\
1<4 \\
(=2=3)\end{array}$ \\
\hline$F$ & 19,82 & 82,93 & 131,07 & 68,33 & & & & \\
\hline dll & $1 / 115$ & $1 / 88$ & $1 / 58$ & $1 / 56$ & & & & \\
\hline sig. & .000 & .000 & .000 & .000 & & & & \\
\hline
\end{tabular}


Tableau 8: Statistiques descriptives et test de normalité des distributions pour les distances aux clusters

\begin{tabular}{|c|c|c|c|c|c|c|c|c|c|c|c|c|}
\hline & & \multicolumn{8}{|c|}{ Statistiques descriptives } & \multicolumn{3}{|c|}{ Tests de normalité } \\
\hline & & Moy & E-t & Min & $\operatorname{Max}$ & Asym & $\begin{array}{c}\text { Er- } \\
\text { Stand } \\
\text { (asym) }\end{array}$ & Aplat & $\begin{array}{c}\text { Er-Stand } \\
\text { (aplat) }\end{array}$ & $\begin{array}{l}\text { Shapiro- } \\
\text { Wilk }\end{array}$ & ddl & Sig \\
\hline \multirow{4}{*}{$\begin{array}{l}\text { Distances } \\
\text { aux } \\
\text { clusters } \\
\text { attribués }\end{array}$} & E1 & 4,90 & 2,07 & 1,0 & 10,0 & 0,33 & 0,37 & 0,80 & 0,73 & 0,942 & 40 & 0,040 \\
\hline & E2 & 4,07 & 1,64 & 1,0 & 8,0 & 0,37 & 0,35 & 0,01 & 0,70 & 0,921 & 45 & 0,005 \\
\hline & E3 & 3,23 & 1,33 & 0,0 & 5,0 & $-1,06$ & 0,43 & 0,83 & 0,83 & 0,876 & 30 & 0,002 \\
\hline & E4 & 3,97 & 2,29 & 0,0 & 8,0 & $-0,22$ & 0,43 & $-0,93$ & 0,85 & 0,935 & 29 & 0,073 \\
\hline \multirow{4}{*}{$\begin{array}{l}\text { Distances } \\
\text { aux autres } \\
\text { clusters }\end{array}$} & $E 1$ & 6,50 & 2,49 & 1,0 & 10,0 & $-0,48$ & 0,34 & $-0,45$ & 0,73 & 0,941 & 40 & 0,038 \\
\hline & E2 & 7,24 & 1,67 & 3,0 & 10,0 & $-0,25$ & 0.35 & $-0,01$ & 0,70 & 0,950 & 45 & 0,051 \\
\hline & E3 & 7,74 & 1,69 & 5,0 & 10,0 & $-0,03$ & 0,43 & $-1,30$ & 0,83 & 0,915 & 30 & 0,020 \\
\hline & E4 & 8,52 & 1,88 & 3,0 & 10,0 & $-1,34$ & 0,43 & $-1,41$ & 0,85 & 0,786 & 29 & 0,000 \\
\hline
\end{tabular}

Comparaison des résultats des études 1 et 2

La comparaison des distances obtenues grâce aux tâches 3 et 4 pour les deux méthodes est fournie dans la figure 5 .

Cette opération montre que globalement la concordance entre les deux méthodes oscille autour de $23 \%$ ou $60 \%$. Il faut souligner que ce recouvrement est significatif $\left(\mathrm{X}^{2}(15)=110,04 ; \mathrm{p}<.001\right)$ et relativement fort $\left(\mathrm{V}_{\mathrm{c}}=0,450 ; \mathrm{p}<.001\right)$.

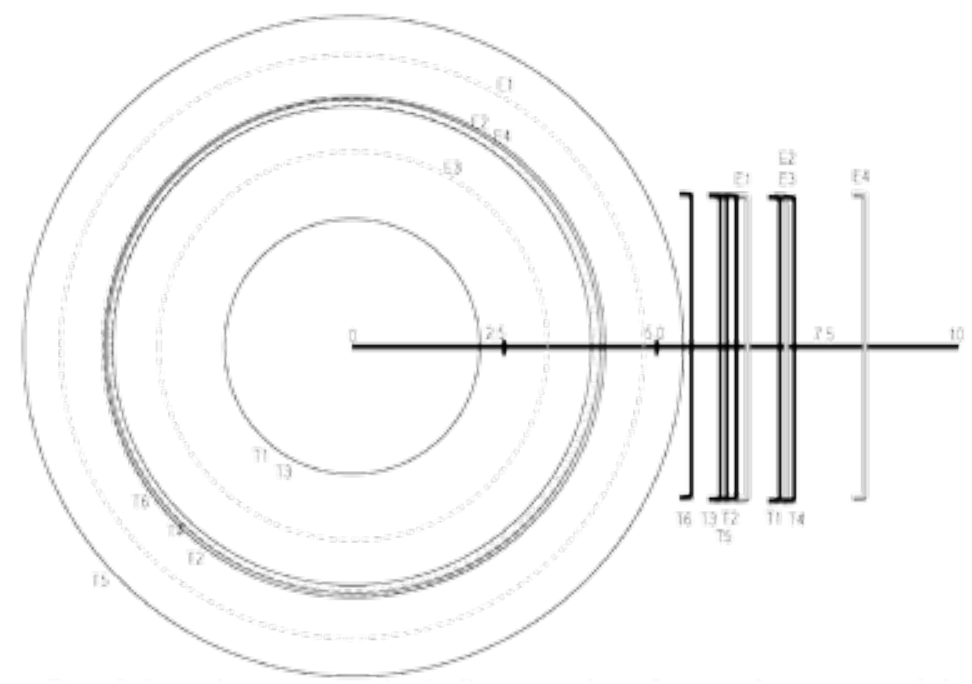

Figure 5: Comparaison, par superposition, des distances aux clusters de types ou de groupes: en cercle, les proximités au cluster attribué, en segment, les distances aux autres clusters; traits pleins etlou noirs pour les types et pointillés gris pour les regroupements 
La figure 5 montre que la méthode par description de types spontanés aboutit à des distances fort amples entre les différents élèves qui y sont associés tout en ayant des distances plus proches avec les clusters auxquels ils ne sont pas associés (traits noirs). La méthode par regroupements obtient des distances prototypiques plus resserrées que la méthode par description de types mais qui restent relativement importantes (traits gris pointillés). Elle permet également une frontière plus nette entre la centralité à un cluster et l'éloignement à un autre cluster.

\section{Conclusion et discussion}

D'un point de vue conceptuel, nos deux analyses en clusters aboutissent à des catégorisations proches. Néanmoins, elles ne sont évidentes que dans deux cas: celui des perturbateurs inattentifs peu performants (T1) et des agités peu performants (E2) d'un côté et, celui des bons élèves scolaires et attentifs (T6) et des bons élèves volontaires (E3). L'association de nos catégories avec celles mises en évidence dans la recherche de Kagan et Tippins (1991) est quasiment nette car elle renvoie, d'une part, à des élèves modèles non problématiques (T6 et E3) correspondant aux bons élèves de Thelen (1967) et, d'autre part, à des élèves perturbateurs ( $\mathrm{T} 1$ et $\mathrm{E} 2$ ) correspondant aux mauvais élèves de Thelen. Le système de catégorie de Thelen (1967) renferme un groupe d'élèves perdus proche de nos clusters T5 et E4. Concernant E1 et T2, parce qu'ils partagent des caractéristiques à l'intersection de plusieurs typologies, il est difficile de le positionner. Enfin, les profils regroupant des élèves moyens tels que T3 et T4 ne sont pas recensés dans les recherches précédemment citées.

Le recouvrement conceptuel est encourageant pour nos deux méthodes qui aboutissent cependant à des catégorisations statistiquement identiques que dans $23 \%$ à $60 \%$ des cas. L'appréciation des taux de recouvrement statistiques est complexe car il n'existe pas de seuil fixé par des chercheurs. Nous osons simplement noter qu'il ne nous satisfait pas car nous nous attendions à un meilleur rapport compte tenu du rapprochement sémantique des deux catégorisations. Deux explications peuvent être avancées: la potentielle modification du profil des élèves entre les tâches qui se sont déroulées sur un maximum de deux semaines pour chaque enseignante (hypothèse peu probable) ou bien, les méthodes saisissent des réalités cognitives différentes. En effet, il est probable que l'approche par descriptifs fasse apparaître des représentations fortement abstraites auxquelles il est plus difficile d'associer des élèves bien réels qui sont probablement regroupés selon des critères situés, multivariés et chronologiquement mobiles. D'un point de vue méthodologique, il s'agira donc, à l'avenir, d'étudier si ces approches mesurent les mêmes entités cognitives.

Concernant la structure centrale-périphérique des cognitions des enseignantes sur les élèves, deux analyses nous ont permis de nous positionner. Premièrement, l'application d'un échelonnement multidimensionnel sur les 
estimations de distance perçues entre les différents types d'élèves permet de supposer une organisation des cognitions en ensembles plus ou moins isolés. D’après les schémas bidimensionnels obtenus, les cognitions des enseignantes sur les élèves sont compartimentées en zones délimitées par des composantes attentionnelle et comportementale pour l'approche des descriptifs, ou par des composantes applicationnelle et de performance pour l'approche par regroupements. Ces composantes renvoient aux critères de Morine-Dershimer (1978) et Kagan et Tippins (1991). À ce stade, on pourrait considérer les cognitions des enseignantes comme des catégories isolées, mais la suite de l'analyse nous montre autre chose.

Ainsi, les moyennes de distance de chaque élève aux clusters permettent de supposer une catégorisation des élèves à l'intérieur des profils identifiés par l'analyse en clusters. Nos tests statistiques montrent toutefois que les classifications à l'intérieur de certains profils sont plus unanimes qu'au sein de certains autres. L'analyse des distributions de distances permet d'estimer qu'il existe des élèves qui sont des représentants parfaits des clusters d'élèves identifiés, et que certains autres élèves sont plus éloignés du prototype de leur cluster pour adopter des valeurs proches des prototypes des autres clusters. Les frontières entre les clusters ne sont donc pas hermétiques. La classification de certains élèves se fait en termes de conditions nécessaires et suffisantes; alors que pour d'autres, la classification est plus incertaine puisqu'elle adopte des valeurs intermédiaires entre plusieurs clusters.

Ces résultats contribuent significativement à la compréhension de la théorie sur l'organisation interne des cognitions des enseignants. Nos résultats vont dans le sens des différentes théories en la matière: organisation typologique en clusters prototypiques, existence d'exemplaires des prototypes, structure centrale-périphérique autour du prototype du cluster et, association en termes de distance aux prototypes. L'association aux prototypes de clusters n'étant pas toujours évidente pour certains élèves, nous rejoignons la théorie de Green (1971) qui accepte une certaine perméabilité des frontières entre clusters compte tenu de l'existence de cognitions isolées au sein de chaque cluster.

Une autre observation concerne les caractéristiques utilisées par les enseignantes pour catégoriser les élèves. Nos analyses montrent que nos enseignantes décrivent les types d'élèves essentiellement à l'aide d'éléments comportementaux ou de traits de personnalité. Si ces éléments ne nous semblent pas dénués de sens pour l'interaction en classe, on peut toutefois s'étonner de la faible utilisation de descripteurs associés à la compréhension scolaire. Une hypothèse peut être avancée pour expliquer ce fait pour l'approche par descriptifs. Pour l'étude 1, nous avons demandé aux enseignantes de décrire des types d'élèves rencontrés au cours de leur carrière. Elles ont probablement décrit des types d'élèves abstraits. Nous aurions peut-être obtenu des données différentes en leur demandant de décrire des types d'élèves de leur classe. 
D'autres limites telles que la taille et la représentativité de notre échantillon peuvent aussi être avancées pour cette étude. L'ensemble des données et interprétations s'appuient sur le concours de neuf enseignantes du canton de Genève. Aussi, une investigation plus large de la population enseignante du canton de Genève est en cours. Ce sont au total 41 enseignants ( 23 du primaire et 18 du secondaire) qui participent à ce projet subventionné par le fonds national suisse. Le design de recherche présenté dans cet article a été répliqué, et complété notamment par des observations en classe afin de confronter la structure des cognitions des enseignants sur les élèves à ce qu'il se passe en situation réelle de classe. Les répercussions pour la recherche sont donc multiples. Il conviendrait d'analyser quels clusters d'élèves sont utilisés par les enseignants pour quels types de décisions pédagogiques. Quels élèves - ceux proches de leur cluster ou ceux qui s'en éloignent - sont pris en compte par les enseignants pour gérer la classe ou pour choisir le rythme à donner à l'enseignement? Mais aussi, dans quelle mesure les enseignants utilisent une typologie basée sur des éléments de compréhension pour asseoir leurs choix pédagogiques en matière de gestion des apprentissages ou une typologie basée sur les comportements et l'attention pour motiver leurs décisions en matière de gestion de la discipline. Il s'agirait également de comparer des enseignants qui démontrent d'excellentes compétences diagnostiques comparativement à des enseignants qui éprouvent des difficultés en la matière (les outils développés par l'équipe de COACTIV permettraient de distinguer ces deux types d'enseignants - voir Brunner, Anders, Hachfeld \& Krauss, 2011). En plus d'un nouvel éclairage porté sur notre thématique de recherche, nous espérons avoir des résultats à niveau de preuve plus élevé en utilisant une méthode plus confirmatoire pour l'analyse des données, analyses que nous n'avons pas pu entreprendre ici au vu de notre échantillon limité de participants. Mais malgré ce design, il faudrait sonder d'autres cantons de la Suisse, voir au national et d'autres pays pour pouvoir généraliser.

De ces observations découle une répercussion évidente pour la formation initiale ou continue des enseignants. En effet, l'analyse des indices sur lesquels se basent des enseignants présentant des compétences diagnostiques optimales pourrait aboutir à des outils d'observation de la compréhension des élèves lors de l'enseignement auxquels les enseignants en formation pourraient être sensibilisés. La comparaison entre l'exactitude du diagnostic et les performances des élèves d'enseignants ayant été formés à ces outils comparativement à des enseignants qui n’y auraient pas été sensibilisés pourrait alors être envisagée. Il s'agit d'un champ qui mériterait l'attention des chercheurs en sciences de l'éducation car la recherche tend à montrer que l'exactitude des diagnostics des enseignants pourrait être améliorée (Praetorius, Lipowsky \& Karst, 2012 ; Südkamp, Kaiser \& Möller, 2012). Puisque les compétences diagnostiques des enseignants jouent un rôle important dans la qualité des apprentissages des élèves (Hattie, 2009), des modules de formation au diagnostic en situation devraient être élaborés sur base de ces résultats. 


\section{Note}

1 Les cinq champs sémantiques retenus sont le fruit d'une analyse de contenu (Bardin, 1977; Richards, 2005) de l'ensemble des descriptions ou groupements produits par les enseignantes. Cette analyse de contenu est menée de concert par trois des auteurs de cet article. Concrètement, pour chaque descriptif ou groupement, les expressions utilisées par les enseignantes sont classées selon leur champ sémantique et selon la valeur positive ou négative des expressions. Par exemple, pour le champ sémantique "comportement», nous distinguons des adjectifs positifs comme calme ou discret, et négatifs comme perturbateur ou agressif. Chaque expression ayant obtenu un poids de la part des enseignantes, nous avons additionné les poids de chaque expression rangés dans les pôles négatif et positif des champs sémantiques pour obtenir 10 sous-scores. Les sous-scores de caractéristiques positifs et négatifs de chaque champ sémantique sont soustraits pour obtenir les cinq scores que nous utilisons pour l'analyse en clusters. La raison de la soustraction réside dans le fait que certains sous-scores obtenaient la valeur de zéro marquant l'absence de descriptions; cette absence est considérée comme une donnée manquante dans le programme SPSS et entraîne l'ignorance du descriptif ou de groupement dans l'analyse en clusters. La soustraction permet de conserver tous les descriptifs ou groupements dans l'analyse, tout en tenant compte de la réalité que l'absence d'un sous-score par exemple positif n'entraîne pas forcément l'absence d'un sous-score négatif; toutes les descriptions ou tous les groupements sont ainsi conservés lors de l'analyse.

\section{Bibliographie}

Abric, J.-C. (2003). La recherche du noyau central et de la zone muette des représentations sociales. In J.-C. Abric (Éd.), Méthodes d'étude des représentations sociales (pp. 59-80). Ramonville Saint-Agne: Erès.

Bardin, L. (1977). L'analyse de contenu. Paris: PUF.

Berliner, D.C. (1987). Ways of thinking about students and classrooms by more and less experienced teachers. In J. Calderhead (Éd.), Exploring teachers' thinking (pp. 60-83). London: Cassell.

Bromme, R. (1989). The "collective student» as the cognitive reference point of teachers' thinking about their students in the classroom. In J. Lowyck \& C.M. Clark (Éd.), Teacher thinking and professional action: Studia paedagogica $n^{\circ} 9$ (pp. 209-222). Leuven, Belgium: Leuven University Press.

Brunner, M., Anders, Y., Hachfeld, A. \& Krauss, S. (2011). Diagnostische Fähigkeiten von Mathematiklehrkräften. In M. Kunter, J. Baumert, W. Blum, U. Klusmann, S. Krauss, \& M. Neubrand (Hrsg.), Professionelle Kompetenz von Lehrkräften (S. 215-234). München: Waxmann.

Calderhead, J. (1983). Research into teachers' and student teachers' cognitions: Exploring the nature of classroom practice. Paper presented at the Annual Meeting of the American Educational Research Association. Montreal, Canada.

Calderhead, J. (1996). Teachers: Beliefs and knowledge. In D.L. Berliner \& R.C. Calfee (Éd.), Handbook of educational psychology (pp. 673-708). New York: MacMillan.

Clatworthy, J., Buick, D., Hankins, M., Weinman, J. \& Horne, R. (2005). The use and reporting of cluster analysis in health psychology: a review. Britsh Journal of Health Psychology, 10, 329-358.

Dahllöf, U.S. (1971). Ability grouping, content validity, and curriculum process analysis. New York: Teachers College Press.

Everitt, B.S., Landau, S., Leese, M. \& Stahl, D. (2011). Cluster Analysis (5th éd.). London: Wiley.

Green, T. (1971). The activities of teaching. New York: McGraw-Hill. 
Hattie, J.A.C. (2009). Visible learning: A synthesis of over 800 meta-analyses relating to achievement. London: Routledge.

Hattie, J.A.C. (2012). Visible learning for teachers: Maximizing impact on learning. London: Routledge.

Hofer, M. (1981). Die Schülerspezifität in Einstellungen und Verhaltensweisen des Lehrers. In M. Haidl (Hrsg.), Lehrerpersönlichkeit und Lehrerrolle im sozial-integrativen Unterricht (S. 58-82). München: Lurz.

Hofer, M. (1986). Sozialpsychologie erzieherischen Handelns: Wie das Denken und Verhalten von Lehrern organisiert ist. Göttingen: Hogrefe.

Hörstermann, T., Krolak-Schwerdt, S. \& Fischbach, A. (2010). Die kognitive Repräsentation von Schülertypen bei angehenden Lehrkräften - Eine typologische Analyse. Revue Suisse des Sciences de l'Education, 32(1), 143-158.

Howell, D.C. (1998). Méthodes statistiques en sciences humaines. Bruxelles: De Boeck.

Kagan, D.M. \& Tippins, D.J. (1991). How student teachers describe their pupils. Teaching and Teacher Education, 7(5/6), 455-466.

Mayer, D. \& Marland, P. (1997). Teachers' knowledge of students: a significant domain of practical knowledge? Asia-Pacific Journal of Teacher Education, 25(1), 17-34.

Medin, D.L. \& Rips, L.J. (2005). Concepts and Categories: Memory, Meaning, and Metaphysics. In K.J. Holyoak \& R.G. Morrison (Éd.), The Cambridge Handbook of Thinking and Reasoning (pp. 37-72). New York: Cambridge University Press.

Morine-Dershimer, G. (1978). How teachers «see» their pupils. Educational Research Quarterly, 3(4), 43-52.

Murphy, G.L. (2010). What are categories and concepts? In D. Mareschal, P.C. Quinn, \& S. Lea (Éd.), The making of human concepts. Oxford: Oxford University Press.

Oldenbürger, H. A. (1986). Does a tendency to group pupils on attributes exist in teachers' cognitions/judgements? In M. Ben Peretz, R. Bromme, \& R. Halkes (Éd.), Advances of research on teacher thinking (pp. 186-200). Lisse: Swets \& Zeitlinger B.V.

Praetorius, A.-K., Lipowsky, F. \& Karst, K. (2012). Diagnostische Kompetenz von Lehrkräften: Aktueller Forschungsstand, unterrichtspraktische Umsetzbarkeit und Bedeutung für den Unterricht. In R. Lazarides \& A. Ittel (Hrsg.), Differenzierung im mathematisch-naturwissenschaftlichen Unterricht: Implikationnen für Theorie und Praxis (S. 115-146). Bad Heilbrunn: Klinkhardt.

Reed, S.K. (2011). Cognition: Théories et applications. Bruxelles: De Boeck.

Richards, L. (2005). Handling qualitative data: a practical guide. London: Sage.

Rosch, E. (1978). Principles of categorization. In E. Rosch \& B.B. Lloyd (Éd.), Cognition and categorization (pp. 27-48). Hillsdale, NJ: Erlbaum.

Roussiau, N. \& Bonardi, C. (2001). Les représentations sociales: état des lieux et perspectives. Sprimont: Mardaga.

Sternberg, R.J. (2007). Manuel de psychologie cognitive: du laboratoire à la vie quotidienne. Bruxelles: De Boeck.

Südkamp, A., Kaiser, J. \& Möller, J. (2012). Accuracy of teachers' judgments of students' academic achievement: a meta-analysis. Journal of Educational Psychology, 104(3), 743-762.

Thelen, H.A. (1967). Classroom grouping for teachability. New York: John Wiley \& sons, inc.

Veyrac, H. \& Blanc, J. (2014). Etude de la catégorisation des élèves par les professeurs Convention d'étude et de recherche, Appel d'offre "égalité des chances à l'école ". Toulouse: Université de Toulouse.

Wanlin, P. (2009). La pensée des enseignants lors de la planification de leur enseignement. Revue Française de Pédagogie, 166, 89-128.

Wanlin, P., \& Crahay, M. (2012). La pensée des enseignants pendant l'interaction en classe. Education et Didactique, 6(1), 1-39. 
Mots-clés: Catégories d'élèves, cognitions enseignantes, structure, enseignement primaire, analyse en clusters

\section{Schülerkategorisierung: eine Studie über die interne Struktur der Lehrerkognitionen über Schülerinnen und Schüler}

\section{Zusammenfassung}

Dieser Beitrag beschreibt eine Analyse der inneren Struktur der Lehrerkognitionen über Schülerinnen und Schüler. Bei der Datenerhebung wurden zwei Methoden der Schülerkategorisierung angewendet: Die Erste nutzt Beschreibungen typischer Schülerinnen und Schülern und die Zweite eine Aufgabe, in der die Lehrpersonen ihre Schülerinnen und Schüler gruppieren mussten. Neun Genfer Grundschullehrpersonen nahmen an der Studie teil. Die Analyse der Daten anhand der Theorien über die interne Kognitionsstrukturen ergibt, dass das Wissen der Lehrpersonen über Schülerinnen und Schüler n der Form von Clustern mit Prototypen und Peripherieelementen schematisiert werden kann. Da die Zuweisung der Schülerinnen und Schüler zu einem Cluster nicht immer eindeutig ist, wird an die Theorie von Green (1971) angeknüpft, welche durchlässige Grenzen zwischen Kategorien vermutet.

Schlagworte: Schülerkategorisierung, Kognitionen von Lehrkräfte, Struktur, Grundschule, Clusteranalyse

\section{Classificazione degli allievi: uno studio sulla struttura interna delle cognizioni degli insegnanti sugli allievi}

Riassunto

Questo contributo propone di studiare la struttura interna delle cognizioni degli insegnanti sugli allievi tramite due metodi di classificazione: quello per descrizione e quello per raggruppamento. Alla luce delle teorie relative all'organizzazione delle cognizioni, la nostra ricerca, condotta su nove insegnanti della scuola elementare di Ginevra, mostra un'organizzazione delle cognizioni degli insegnanti in cluster secondo una struttura centrale-periferica. Dato che l'associazione ai prototipi di cluster non è ancora del tutto chiara per alcuni allievi, facciamo riferimento all'idea di isolamento tra le cognizioni formulata da Green (1971) che accetta una certa permeabilità tra i confini dei cluster.

Parole chiave: Classi di allievi, cognizioni insegnanti, struttura, insegnanti primaria, analisi in cluster 


\section{Students' categorization: a study on the internal structure of teachers' cognitions about students}

\section{Summary}

This paper suggests a study on the internal structure of teachers' cognitions about students using two methods of students' categorization: the first one uses description of typical students and the second a student grouping task. Data gathered through the participation of nine Geneva elementary school teachers and analyzed in the light of theories about the structure of cognitions shows that teachers' knowledge about students adopt a clusterized shape comprising prototypes and peripheral elements. As students' association to a cluster isn't always clear-cut, we converge with Green's (1971) theory that the boundaries between student categories are permeable.

Keywords: Students' categorization, teachers cognitions, structure, primary school, cluster analysis 